There has been a reduction in variation between acute trusts in the number of admissions and length of stay (without an increase in re-admission rate). We believe our COPD Dashboard has helped drive this change.

\section{P31 INTELLIGENCE BASED INFORMATION SYSTEM (IBIS) REDUCES RESPIRATORY PATIENTS' USE OF SECONDARY HEALTH CARE RESOURCES}

${ }^{1}$ SKM Harlow, ${ }^{2}$ T Tollit, 'MJ Irvin-Sellers. 'Ashford and St Peter's Hopsitals NHS Foundation Trust, Chertsey, UK; ${ }^{2}$ Respiratory Care Team North West Surrey, VirginCare, Chertsey, UK

\subsection{6/thoraxjnl-2014-206260.181}

Background IBIS is a database developed by South East Coast Ambulance Service (SECAmb) to facilitate communication of individual patient care plans between SECAmb, the Respiratory Care Team (RCT) and secondary care. It aims to reduce the number of patients conveyed to hospital.

Aims and objectives The investigation aimed to establish the impact of IBIS on respiratory patients' use of secondary care in our locality.

Methods Respiratory patients uploaded into IBIS between May and November 2013 were included. Data were collected from the Patient Administration System including, number of A\&E attendances and admissions in the three months preceding and three months after patient care plans were included in IBIS. Data were analysed with descriptive statistics and Wilcoxon Paired Test utilising SPSS version 22.

Results 65 patients were included in the study. Table 1 demonstrates the impact of IBIS on A\&E attendances and admissions. There was a significant reduction in admissions $(\mathrm{p}=0.011)$. A reduction in $\mathrm{A} \& \mathrm{E}$ attendances was observed $(p=0.064)$. A sub-analysis of patients already utilising secondary care resources was undertaken. In this patient group a significant reduction in both $\mathrm{A} \& \mathrm{E}$ attendances $(\mathrm{p}=$ $0.000)$ and admissions $(p=0.000)$ was observed.

Conclusions IBIS assists in reducing respiratory patients A\&E attendances and admissions. The impact of IBIS is more profound in patients who have already utilised secondary health care resources.

\begin{tabular}{llc}
\hline Abstract P31 Table 1 & Median (range) & $\mathrm{p}=$ \\
\hline $\mathrm{n}=$ A\&E attendances & $1(0$ to 12$)$ & 0.064 \\
3 months pre IBIS & \\
$\mathrm{n}=$ A\&E attendances & $0(0$ to 6$)$ & \\
3 months post IBIS & \\
$\mathrm{n}=$ Admissions & $0(0$ to 12$)$ & 0.011 \\
3 months pre IBIS & \\
$\mathrm{n}=$ Admissions & $0(0$ to 2$)$ & \\
3 months post IBIS &
\end{tabular}

P32 A NOVEL AUTOMATED REFERRAL SYSTEM USING THE ELECTRONIC PRESCRIPTION OF PREDNISOLONE $\geq 30$ MG AND NEBULISED BRONCHODILATORS TO THE RESPIRATORY SPECIALIST TEAM IS ROBUST AND EFFECTIVE

RC Colclough, T Avent, K Breese, C Craddock, D Curry, K Swindells, S Gompertz. Queen ElizabethHospitalBirmingham, Birmingham, UK

10.1136/thoraxjnl-2014-206260.182

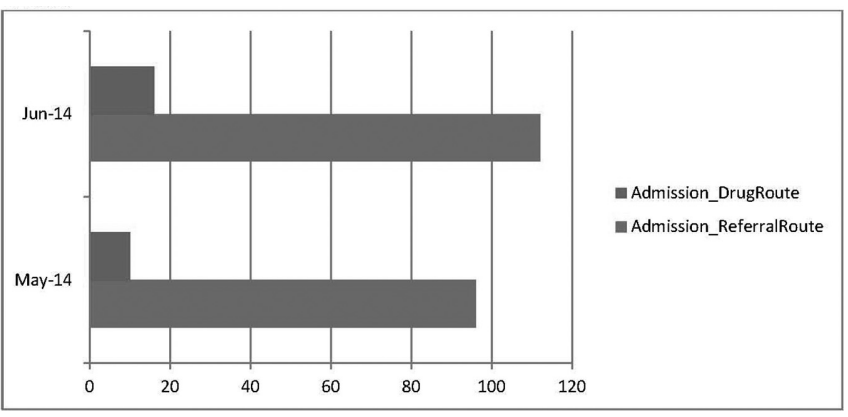

Abstract P32 Figure 1 Average time difference from time of admission to time of referral/ Drug route (Automated email) in hours

Introduction Patients admitted to hosptial with an exacerbation of COPD should be cared for by respiratory teams (COPD Quality Standard 10, NICE 2011). The earlier the patient is reviewed by a specialist the greater the impact on length of stay (COPD NICE guideline 101, 2010). A rigorous and rapid referral system is required.

A new electronic referral system triggered by the prescription of prednisolone $>=30 \mathrm{mg}$ AND nebulised bronchodilators (salbutamol and/or ipratropium) via our Prescribing Information Communication System (PICS) was implimented. This replaced the laborious paper sift of the admissions book for admissions with airway exacerbations. The general medical team was also permitted to refer directly to the respiratory team via email.

Referral numbers were compared over a 2 -month period to ensure that the new automated system is robust.

Method

1. The new automated referral was created.

2. Data was collected from the three referral routes a) paper sift, b) automated referral system, c) email from general medical team.

3. Comparison between: a) monthly automated and email referrals was made, b) paper sift and automated referrals route was made.

Results Each month there were:

1. 262 (mean) admissions screened via paper sift of which 96 (mean) were inappropriate (36\%).

2. No patients identified by paper sift or email were missed by the automated system.

3. 138 (mean) automated referrals- time from admission to automated referral $13 \mathrm{~h}$ (mean) 10-16 h (range).

4. 75 (mean) email referrals - time from admission to email referral $104 \mathrm{~h}$ (mean)- 96-112 (range).

Conclusion Paper sift is time costly and laborious with a third of referrals inappropriate. Automated referrals are sent 91 (mean) hours quicker than emai referrals. Automated referrals reduce the delay between admission and specialist review. They can be received from any location in the hospital throughout the day using Smart Phones.

The automated referral eliminates the need for once daily paper sifting of the admission book, and replaces it with a more timely and robust method of directing the specialist respiratory team to the patient's bedside. 